\title{
Evaluation of Influenza A H1N1 infection and antiviral utilization in a tertiary care hospital
}

\author{
Talita Rantin Belucci ${ }^{1,2^{*}}$ (D), Alexandre R. Marra ${ }^{2,3}$, Michael B. Edmond ${ }^{3,4}$, João Renato Rebello Pinho ${ }^{5,8}$, \\ Paula Kiyomi Onaga Yokota ${ }^{2}$, Ana Carolina Cintra Nunes Mafra ${ }^{6,7}$ and Oscar Fernando Pavão dos Santos ${ }^{2}$
}

\begin{abstract}
Background: Influenza A H1N1 infections carry a significant mortality risk. This study describes inpatients with suspected and confirmed Influenza A H1N1 infection who were prescribed oseltamivir, the risk factors associated with infection, the association between infection and mortality, and the factors associated with in-hospital mortality in infected patients.

Methods: This study was a matched case-control study of hospitalized patients who underwent real-time polymerase chain reaction testing for Influenza A H1N1 and were treated with oseltamivir from 2009 to 2015 in a tertiary care hospital. Cases (patients with positive Influenza A H1N1 testing) were matched 1:1 to controls (patients with negative test results).

Results: A total of 1405 inpatients who underwent PCR testing and received treatment with oseltamivir were identified in our study and 157 patients confirmed Influenza A H1N1. Almost one third of patients with Influenza A H1N1 were diagnosed in the pandemic period. There was no difference in mortality between cases and controls. Immunocompromised status, requirement of vasoactive drugs, mechanical ventilation, acute hemodialysis, albumin administration, surgical procedures and thoracic procedures and length of stay were associated with increased risk of death in Influenza A H1N1 infected patients.
\end{abstract}

Conclusions: We found no increased risk of mortality for patients with proven Influenza A H1N1 when compared to similar patients without confirmed Influenza.

Keywords: Influenza a H1N1, Oseltamivir, Hospitalized patients

\section{Background}

According to the World Health Organization (WHO), during the Influenza A H1N1 pandemic 59 million people were infected, resulting in 265,000 hospitalizations and 12,000 deaths in the United States. This virus has high transmissibility, a short incubation period, and high rates of morbidity and mortality [1].

The goal of this study is to describe inpatients treated with oseltamivir and suspected and confirmed Influenza A H1N1 infection, the associated factors with infection,

\footnotetext{
* Correspondence: talita.belucci@hotmail.com

${ }^{1}$ Hospital Israelita Albert Einstein, São Paulo, Brazil

${ }^{2}$ Division of Medical Practice, Hospital Israelita Albert Einstein, Avenida Albert Einstein, 627 - bloco A1, $1^{\circ}$ andar, Morumbi, São Paulo 05651-901, Brazil Full list of author information is available at the end of the article
}

the association between infection and mortality, and the factors associated with in-hospital mortality in patients with confirmed Influenza A H1N1.

\section{Methods}

This study was conducted in a tertiary care, private hospital in São Paulo, Brazil with 629 beds and approximately 194,000 patient-days yearly and approved by the Institutional Review Board and Ethics Committee of Hospital Israelita Albert Einstein and informed consent was not required.

A retrospective study was conducted from January 2009 to December 2015.

This study describes inpatients treated with oseltamivir who had suspected or confirmed Influenza A H1N1

(c) The Author(s). 2018 Open Access This article is distributed under the terms of the Creative Commons Attribution 4.0 International License (http://creativecommons.org/licenses/by/4.0/), which permits unrestricted use, distribution, and 
infection and were tested for Influenza A H1N1 by real-time polymerase chain reaction (RT-PCR). The primary reason for hospitalization was not necessarily Influenza. This study also describes the factors associated with infection, the association between infection and mortality and the factors associated with in-hospital mortality in patients with confirmed Influenza A H1N1.

A matched (1:1) case-control study was performed to analyze the factors associated with infection and in-hospital mortality, comparing patients who would have similar illness severity during hospitalization and thus isolate the impact of the infection on the outcome of in-hospital mortality. Cases were defined as patients with Influenza A H1N1 confirmed by RT-PCR, and controls had a negative result for Influenza A (H1N1 and H3N2) and Influenza B and were treated with oseltamivir for up to four days. All patients in the matched case-controls study were tested both for Influenza A H1N1 and Influenza A H3N2, but only 31.6\% (444/1.405) were tested for Influenza B. Patients excluded were those under 18 years of age and those in whom the length of stay exceeded 365 days. The criteria of length of stay was based on the long-term hospitalized patients.

The data abstracted from the electronic medical record included demographics and clinical data, Influenza RT-PCR assay results, oseltamivir treatment (duration, frequency and dose), outcome status (death was defined as in-hospital mortality), and underlying conditions (lung disease, cardiovascular disease, neurological and neuro-developmental conditions, blood disorders, diabetes mellitus, kidney disease, liver disease, immunosuppression (e.g., HIV, cancer or chronic treatment with corticosteroids), and pregnancy or post-partum state [up to two weeks after childbirth]). We also collected possible indicators of complications during hospitalization (which may or may not be associated with H1N1 infection): data on intensive care unit (ICU) admission, transfusions, use of mechanical ventilation, acute hemodialysis, use of vasopressor drugs, albumin and antibiotic administration, surgical procedures, or thoracic procedures (e.g., pulmonary biopsy or segmentectomy, tracheostomy).

Patients were not followed up after hospital discharge. Antiviral therapy was prescribed according to the institutional protocol [2], with oseltamivir initiated empirically based on clinical presentation or after a positive PCR test. The empiric therapy for Influenza is based on the symptoms, such as fever, cough, sore throat, runny and/or stuffy nose, muscle or body aches, headaches, and fatigue, as well as for patients at high risk for developing Influenza- related complications. This includes age $\geq 60$ years, patients of any age with certain chronic medical conditions (such as cardiovascular disease, lung disease, diabetes mellitus, kidney disease, liver disease, neurological and neuro-developmental conditions, and immunocompromised states), and pregnant women or post-partum state $[1,2]$.

\section{Statistical analysis}

Descriptive analysis was performed using the median and inter-quartile range (IQR) for continuous variables and absolute frequencies and percentages for categorical variables. Simple associations were analyzed using logistic models and odds ratio were determined. The level of significance was set at 0.05 .

For matching cases and controls we used the Matching package [3], which weighs all variables involved in order to have a balanced final pairing. Cases and controls were matched on factors impacting mortality: age, ICU admission, surgical procedure, use of vasoactive drugs, use of mechanical ventilation, albumin administration, and blood or platelet transfusion. After matching, the logistic model predicting death was adjusted by means of generalized estimation equations, with the Geepack package [4]. We used R software version 3.4.1.

\section{Results}

Of 1,405 inpatients who underwent PCR testing and received treatment with oseltamivir, 1051 (74.8\%) were PCR negative. Twenty-two patients were positive for Influenza B, 175 positive for Influenza A H3N2, and 157 positive for Influenza A H1N1. Of the uninfected patients, 642 received oseltamivir treatment up to four days and 157 of those were matched as controls. For the 157 controls, 32 (20.4\%) underwent Influenza B testing and were also negative for Influenza B (Fig. 1).

When considering the entire period of the study, 19.2\% of the requests for PCR tests combined with oseltamivir prescriptions occurred during the period of the pandemic, with the majority (60.7\%) occurring between 2013 and 2015 . These occurred in older patients $(55.1 \% \geq 60$ years), $49.9 \%$ males, $1.9 \%$ pregnant or post-partum state, $8.1 \%$ immunocompromised, $23.7 \%$ with diabetes mellitus, $24.3 \%$ with lung disease, $1.2 \%$ with liver disease, $6.0 \%$ with kidney disease, $46.8 \%$ with cardiovascular disease and $6.8 \%$ with neurological and neuro-developmental conditions (Additional file 1: Table S1). The primary diagnosis was a disease of the respiratory system in $66.6 \%$ of hospitalizations (Additional file 1: Table S1).

Of the 157 Influenza A H1N1 cases, 49.7\% (78/157) were diagnosed in the pandemic period. In $85.4 \%(134 /$ 157) of the patients with Influenza A H1N1 infection, the daily dosage of oseltamivir was $150 \mathrm{mg}$ and $92.4 \%$ $(145 / 157)$ were treated for 5-10 days (Table 1$)$.

From 2013, the number of hospitalizations increased, especially in the uninfected group (666/1051) (Table 1). Infected patients were 2.86 fold more likely to be immunocompromised $(P=0.033)$ and one-third less likely to 


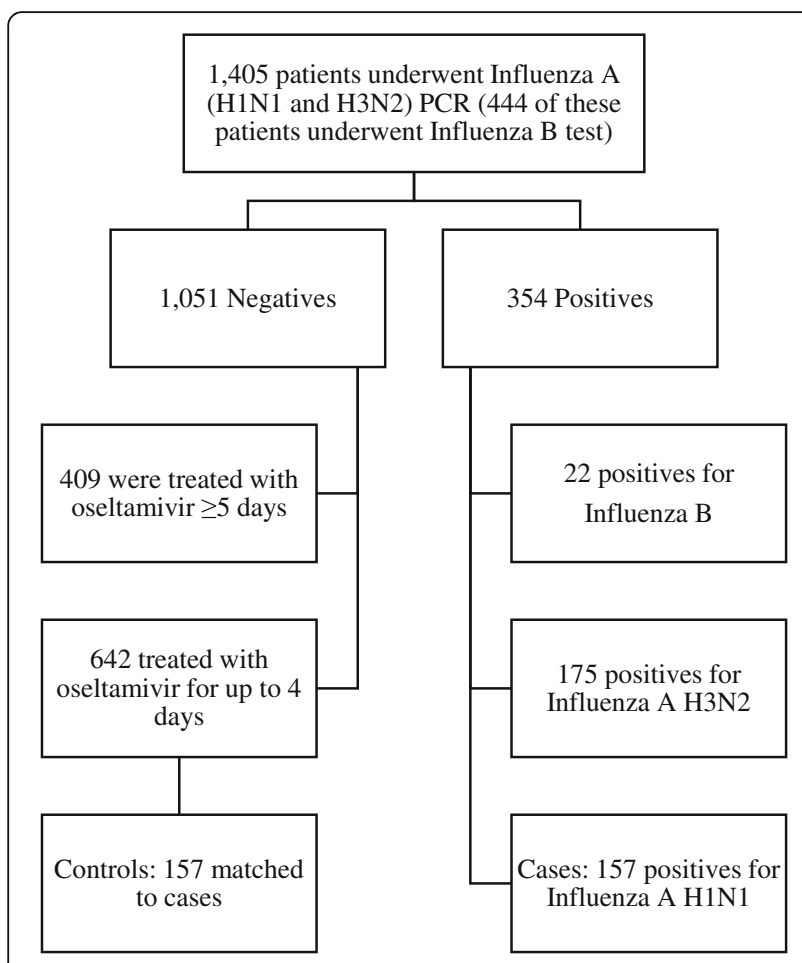

Fig. 1 Patients included in the matched (1:1) case-control study

receive antibacterial therapy $(P=0.005)$ when compared to similar patients without confirmed Influenza (Table 2).

Table 2 also shows for those patients with Influenza A H1N1 infection, 86\% (135/157) were also treated with antibacterials. The median of length of stay was 5 days, and $39.5 \%(62 / 157)$ of the patients were admitted to the

Table 1 Characteristics of inpatients with suspected Influenza $(N=1208)$

\begin{tabular}{lll}
\hline \multirow{2}{*}{ Variable } & \multicolumn{2}{l}{ Influenza status } \\
\cline { 2 - 3 } & $\begin{array}{l}\text { Infected } \\
(n=157)\end{array}$ & $\begin{array}{l}\text { Uninfected } \\
(n=1051)\end{array}$ \\
\hline Period $-n(\%)$ & $78(49.7)$ & $185(17.6)$ \\
2009 (Pandemic period) & $12(7.7)$ & $200(19.0)$ \\
2010 to 2012 & $67(42.7)$ & $666(63.4)$ \\
2013 to 2015 & & \\
Oseltamivir daily dosage $-n$ (\%) & - & $2(0.2)$ \\
75 mg & $134(85.4)$ & $925(88.0)$ \\
150 mg & $23(14.6)$ & $124(11.8)$ \\
300 mg & & \\
Oseltamivir treatment duration (days) $-n(\%)$ & & $642(61.1)$ \\
$<5$ days & - & $405(38.5)$ \\
5 to 10 days & $145(92.4)$ & $4(0.4)$ \\
\hline 10 days & $12(7.6)$ & 4 \\
\hline
\end{tabular}

Category variables presented by absolute and relative frequencies. Numerical variables presented by median and inter-quartile range
ICU. Eight patients (5.1\%) required acute hemodialysis and six patients $(3.8 \%)$ underwent a thoracic procedure (lung biopsy, lung segmentectomy, thoracostomy with closed drainage, drainage of chest wall hematoma, or tracheostomy).

Of patients with Influenza A H1N1, 5.1\% died. Independent factors associated with mortality were requirement for vasoactive drugs (OR $=17.13$, IC 95\%: 5.28 55.59, $P<0.001)$, and length of stay $(\mathrm{OR}=1.03$, IC 95\%: $1.01-1.06, P=0.010)$, and when controlling for these two factors, infection with Influenza A H1N1 was not an independent predictor of mortality ( $\mathrm{OR}=0.45$, CI $95 \%$ : $0.15-1.35, P=0.154$ ) (Table 3).

When considering only the 157 patients with Influenza A H1N1 infection, the associated factors with death were: immunocompromised state $(P=0.019)$, requirement for vasoactive drugs $(P<0.001)$, mechanical ventilation $(P<$ $0.001)$, acute hemodialysis $(P=0.024)$, surgical procedure $(P<0.001)$, thoracic surgery $(P<0.001)$, albumin administration $(P<0.001)$, and length of stay $(P<0.001)$ (Table 4$)$.

\section{Discussion}

We observed a high proportion of negative PCRs among patients treated with oseltamivir because the treatment was administered empirically based on symptoms. The recommendation is to initiate the treatment with oseltamivir within $48 \mathrm{~h}$ of Influenza symptom onset [5], especially in critically ill patients, in order to reduce symptom duration, complications such as pneumonia, and possibly death [6]. However, empiric therapy leads to uninfected patients receiving treatment and testing modalities other than PCR often have low sensitivity and specificity $[7,8]$.

This study considered the RT-PCR test as a gold standard, but other tests, such Influenza A and B by immunofluorescence, Influenza A serology, Influenza B serology, Rapid Influenza A and B diagnostic test, screening for respiratory virus (Influenza $\mathrm{A}$ and $\mathrm{B}$ ) by immunofluorescence, viral culture (Influenza A and B), may have been conducted during the study period.

In our study, surgical procedures and thoracic procedures were associated with an increased risk of death in Influenza A H1N1 patients, but we did not find others studies that analyzed surgical procedures in inpatients with Influenza A H1N1 infection.

More than $80 \%$ of the patients with Influenza A H1N1 in our study were treated with antibacterials, which in some cases was due to pneumonia complicating Influenza. In our study it was not possible to identify if the patients had pneumonia, but in a large cohort study [9] $31 \%$ of the patients with Influenza A H1N1 infection were diagnosed with bacterial pneumonia.

We observed that the number of hospitalizations increased substantially from 2013 onward. This rise can be explained by the fact that in that period a viral panel 
Table 2 Patients profile and associated factors with H1N1 infection based on matched case-control study $(n=314)$

\begin{tabular}{|c|c|c|c|c|}
\hline \multirow{3}{*}{ Variable } & \multicolumn{2}{|l|}{ Influenza status } & \multirow{3}{*}{ OR $(95 \% \mathrm{Cl})$} & \multirow{3}{*}{$P$ value } \\
\hline & Infected & Uninfected & & \\
\hline & $(n=157)$ & $(n=157)$ & & \\
\hline \multicolumn{5}{|l|}{ Characteristics of patients } \\
\hline Male & $85(54.1)$ & $83(52.9)$ & $1.05(0.68-1.64)$ & 0.821 \\
\hline Age (> 60 years old) & $37(23.6)$ & $58(36.9)$ & - & - \\
\hline Cardiovascular disease & $54(34.4)$ & $49(31.2)$ & $1.16(0.72-1.85)$ & 0.548 \\
\hline Lung disease & $31(19.7)$ & $42(26.8)$ & $0.67(0.39-1.14)$ & 0.143 \\
\hline Diabetes mellitus & $28(17.8)$ & $28(17.8)$ & $1.00(0.56-1.79)$ & 1.000 \\
\hline Pregnant or post-partum state & $11(7.0)$ & $4(2.5)$ & $2.88(0.96-10.58)$ & 0.075 \\
\hline Immunocompromised & $16(10.2)$ & $6(3.8)$ & $2.86(1.14-8.15)$ & 0.033 \\
\hline Kidney disease & $11(7.0)$ & $6(3.8)$ & $1.90(0.70-5.63)$ & 0.219 \\
\hline Liver disease & $1(0.6)$ & $3(1.9)$ & $0.329(0.016-2.602)$ & 0.338 \\
\hline Neurological and neuro-developmental conditions & $8(5.1)$ & $11(7.0)$ & $0.71(0.27-1.81)$ & 0.479 \\
\hline \multicolumn{5}{|l|}{ Hospitalization characteristics } \\
\hline Antibacterial therapy (intravenous or oral) & $135(86.0)$ & $150(95.5)$ & $0.29(0.11-0.66)$ & 0.005 \\
\hline ICU stay & $62(39.5)$ & $74(47.1)$ & $0.73(0.47-1.14)$ & 0.172 \\
\hline Mechanical ventilation & $17(10.8)$ & $17(10.8)$ & - & - \\
\hline Acute hemodialysis & $8(5.1)$ & $0(0.0)$ & - & - \\
\hline Vasoactive drugs & $20(12.7)$ & $20(12.7)$ & - & - \\
\hline Red blood cell or platelet transfusion & $4(2.5)$ & $4(2.5)$ & - & - \\
\hline Surgical procedure & $15(9.6)$ & $15(9.6)$ & - & - \\
\hline Thoracic procedure & $6(3.8)$ & $0(0.0)$ & - & - \\
\hline Albumin administration & $23(14.6)$ & $24(15.3)$ & - & - \\
\hline Length of stay (days) & $5.00[3.00 ; 8.00]$ & $5.00[3.00,10.00]$ & $1.00(0.98-1.01)$ & 0.680 \\
\hline In-hospital death & $8(5.1)$ & $14(8.9)$ & $0.55(0.21-1.32)$ & 0.190 \\
\hline
\end{tabular}

Category variables presented by absolute and relative frequencies. Numerical variables presented by median and inter-quartile range. Odds ratio (OR), confidence interval $(95 \% \mathrm{Cl})$ and $p$ value obtained by simple logistic regression

performed by PCR method was introduced in the hospital's testing routine, and beyond Influenza A H1N1, the panel could also identify Influenza A H3N2 and Influenza B. That is why the information on Influenza B is not available for all patients in the study. From 2013 to 2015, the most prevalent Influenza virus in the Southeast region of Brazil, (where the hospital is located) was Influenza A H3N2 [10-12].

In the matched case-controls study results, there was no difference in mortality between patients with and without Influenza infection. However, it should be noted that all

Table 3 Independent predictors of death in inpatients with suspected Influenza A H1N1 $(n=314)$

\begin{tabular}{lll}
\hline Variable & OR $(95 \% \mathrm{Cl})$ & $P$ value \\
\hline Influenza A H1N1 infection & $0.45(0.15 ; 1.35)$ & 0.154 \\
Administration of vasoactive drugs & $17.13(5.28 ; 55.59)$ & $<0.001$ \\
Length of stay (days) & $1.03(1.01 ; 1.06)$ & 0.010 \\
\hline
\end{tabular}

OR odds ratio. Cl: $95 \%$ confidence interval patients were treated with oseltamivir. This finding reinforces the need for treatment within $48 \mathrm{~h}$ of symptom onset, even in those patients who are not at high risk of developing Influenza-related complications $[1,5]$.

In our study, only 8 (5.1\%) patients with Influenza A H1N1 infection died. Immunocompromised states were associated with mortality in patients with Influenza A H1N1 infection in our study and in a Spanish study in which 25\% (68/274) of immunocompromised inpatients with Influenza A H1N1 infection died [13]. A study performed in immunosuppressed patients with Influenza A H1N1 admitted to the ICU concluded that this population has a poor outcome and the use of corticosteroids is strongly discouraged [14].

Patients that require vasoactive drugs and mechanical ventilation were also at increased risk of death from Influenza A H1N1 infection. In the same Spanish study, $78.7 \%$ (214/274) Influenza A H1N1 infected patients requiring vasoactive drugs and $92.2 \%$ (249/274) requiring mechanical ventilation also died [13]. The median length 
Table 4 Univariate predictors of death in patients with confirmed Influenza A H1N1 infection $(n=157)$

\begin{tabular}{|c|c|c|c|c|}
\hline Variable & Discharge $(n=149)$ & Death $(n=8)$ & OR $(95 \% \mathrm{Cl})$ & $P$ value \\
\hline Male gender & $79(53.0)$ & $6(75.0)$ & $2.66(0.59 ; 18.55)$ & 0.240 \\
\hline Age (> 60 years old) & $35(23.5)$ & $2(25.0)$ & $1.09(0.15 ; 4.96)$ & 0.922 \\
\hline Immunocompromised status & $13(8.7)$ & $3(37.5)$ & $6.28(1.18 ; 28.72)$ & 0.019 \\
\hline Diabetes mellitus & $25(16.8)$ & $3(37.5)$ & $2.98(0.58 ; 12.94)$ & 0.153 \\
\hline Kidney disease & $9(6.0)$ & $2(25.0)$ & $5.19(0.69 ; 26.60)$ & 0.063 \\
\hline Cardiovascular disease & $50(33.6)$ & $4(50.0)$ & $1.98(0.45 ; 8.69)$ & 0.348 \\
\hline \multicolumn{5}{|l|}{ Hospitalization characteristics } \\
\hline Vasoactive drugs & $14(9.4)$ & $6(75.0)$ & $28.93(6.04 ; 210.94)$ & $<0.001$ \\
\hline Mechanical ventilation & $12(8.1)$ & $5(62.5)$ & $19.03(4.18 ; 102.52)$ & $<0.001$ \\
\hline Acute hemodialysis & $6(4.0)$ & $2(25.0)$ & $7.94(1.02 ; 44.30)$ & 0.024 \\
\hline Surgical procedure & $10(6.7)$ & $5(62.5)$ & $23.17(5.00 ; 127.25)$ & $<0.001$ \\
\hline Thoracic surgery & $3(2.0)$ & $3(37.5)$ & $29.20(4.49 ; 199.40)$ & $<0.001$ \\
\hline Albumin administration & $17(11.4)$ & $6(75.0)$ & $23.29(4.93 ; 168.04)$ & $<0.001$ \\
\hline Length of stay (days) & $5[3,7]$ & $38[11.5,53.75]$ & $1.07(1.03 ; 1.11)$ & $<0.001$ \\
\hline
\end{tabular}

Category variables presented by absolute and relative frequencies. Numerical variables presented by median and inter-quartile range. $O R$ odds ratio. $95 \% \mathrm{Cl} 95 \%$ confidence interval

of stay for inpatients who died in our study was much higher than that observed in this same study (13 days) [13].

Another Spanish study found that chronic conditions were an independent risk factor for mortality [15]; we noted a similar trend in our study that was not statistically significant.

Our study has some limitations, primarily that it is single center and retrospective. The retrospective nature of the study made it impossible to identify the exact cause of pneumonia. We also cannot attribute the cause of death to Influenza infection. Our study evaluated only patients tested by PCR and were treated with oseltamivir. The patients were not followed after discharge, so it was only possible to identify in-hospital deaths. It was also not possible to check the patient's vaccination status.

\section{Conclusion}

In conclusion, the profiles of the infected and uninfected patients were very similar and there was no difference in mortality. The only risk factor associated with death in infected patients was an immunocompromised state.

\section{Additional file}

Additional file 1: Table S1. Profile of inpatients suspected for Influenza A H1N1 infection, with oseltamivir prescription and underwent real-time polymerase chain reaction (RT-PCR). (DOCX 17 kb)

\section{Abbreviations}

Cl: Confidence intervals; ICU: Intensive care unit; IQR: Inter-quartile range; OR: Odds ratios; RT-PCR: Real-time polymerase chain reaction; WHO: World Health Organization

\section{Acknowledgements}

We gratefully acknowledge the healthcare workers that cared for H1N1 Influenza patients at Hospital Israelita Albert Einstein, São Paulo, Brazil.

Funding

No funding was obtained for this study.

\section{Availability of data and materials}

Confidentially agreements prevent us from sharing the raw data generated during this study. The data available upon request. The corresponding author should be contacted if someone wants to request the data.

\section{Authors' contributions}

TRB, JRRP, PKOY participated in the data collected. TRB, ARM, ACCNM, OFPS participated in the data analysis. TRB, ARM, MBE, OFPS participated in the design and coordination. TRB, ARM, MBE, JRRP, PKOY, ACCNM, OFPS helped to draft the manuscript and to provide critical review to the manuscript. All authors read and approved the final manuscript.

Ethics approval and consent to participate

This study was approved by the Ethics Committee of the Instituto Israelita de Pesquisa Albert Einstein. The requirements for informed consent were waived by our IRB in accordance of the Code of Federal Regulation and of the Privacy Rule.

\section{Consent for publication}

Not applicable.

\section{Competing interests}

The authors declare that they have no competing interests. This research received no specific grant from any funding agency in the public, commercial, or non-for-profit sectors.

\section{Publisher's Note}

Springer Nature remains neutral with regard to jurisdictional claims in published maps and institutional affiliations.

\section{Author details}

${ }^{1}$ Hospital Israelita Albert Einstein, São Paulo, Brazil. ${ }^{2}$ Division of Medical Practice, Hospital Israelita Albert Einstein, Avenida Albert Einstein, 627 bloco A1, $1^{\circ}$ andar, Morumbi, São Paulo 05651-901, Brazil. ${ }^{3}$ Office of Clinical Quality, Safety and Performance Improvement, University of lowa Hospitals

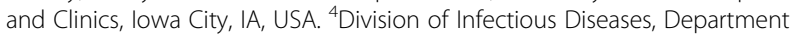


of Internal Medicine, University of lowa Carver College of Medicine, lowa City, IA, USA. ${ }^{5}$ Clinical Laboratory, Hospital Israelita Albert Einstein, São Paulo, Brazil. ${ }^{6}$ Statistics Department, Instituto Israelita de Ensino e Pesquisa Albert Einstein, Hospital Israelita Albert Einstein, São Paulo, Brazil. ${ }^{7}$ Núcleo de Indicadores e Sistemas de Informações, Hospital Israelita Albert Einstein, São Paulo, Brazil. 'LIM 03/07, Faculdade de Medicina da USP, São Paulo, Brazil.

Received: 27 June 2018 Accepted: 1 November 2018

\section{Published online: 16 November 2018}

\section{References}

1. Writing Committee of the WHO Consultation on Clinical Aspects of Pandemic (H1N1) 2009 Influenza. Clinical aspects of pandemic 2009 influenza a (H1N1) virus infection. N Engl J Med. 2010;362(18):1708-19 Review. Erratum in: N Engl J Med 2010 May 27:362(21):2039.

2. Hospital Israelita Albert Einstein Síndrome gripal - diretrizes para diagnóstico e tratamento [institucional protocol]. [2014 Feb 10; updated 2015 Apr 25; Pires EM]

3. Sekhon JS. Multivariate and propensity score matching software with automated balance optimization: the matching package for R. J Stat Softw. 2011;42(7):1-52.

4. Halekoh U, Højsgaard S, Yan J. The R package geepack for generalized estimating equations. J Stat Softw. 2006;15(2):1-11.

5. Rewar S, Mirdha D, Treatment RP. Prevention of pandemic H1N1 influenza. Ann Glob Health. 2015;81:645-53.

6. Coleman BL, Hassan K. Pre-and post-pandemic trends in antiviral use in hospitalized patients with laboratory - confirmed influenza: 2004/05 2013/14, Toronto, Canada. Antivir Res. 2017;140:158-63.

7. McGeer AJ. Diagnostic testing or empirical therapy for patients hospitalized with suspected influenza: what to do? Clin Infect Dis. 2009;48:S14-9.

8. Zazueta-García R, Canizalez-Roman A, Flores-Villaseñor H, Martínez-Garcia J, Llausas-Vargas A, León-Sicairos N. Effectiveness of two rapid influenza tests in comparison to reverse transcription-PCR for influenza a diagnosis. J Infect Dev Ctries. 2014;8:331-8.

9. Shah SN, Greenber JA, McNulty MC, Gregg KS, Riddell J, Mangino JE, et al. Severe influenza in 33 US hospitals, 2013 - 2014: complications and risk factors for death in 507 patients. Infect Control Hosp Epidemiol. 2015; 36:1251-60.

10. Brazil. Ministério da Saúde. Secretaria de Vigilância em Saúde. Influenza: monitoramento até a semana epidemiológica 52 de 2013 [Internet]. Brasília (DF); [201-?] [cited in 2014 May 22]. (Boletim epidemiológico) Available from: http://portalarquivos.saude.gov.br/images/pdf/2014/maio/22/boletiminfluenza-se52de2013-220514.pdf

11. Brazil. Ministério da Saúde. Secretaria de Vigilância em Saúde. Influenza: monitoramento até a semana epidemiológica 28 de 2014 [Internet]. Brasília (DF); [201-?] [cited in 2014 May 22]. (Boletim epidemiológico) Available from: http://portalarquivos2.saude.gov.br/images/pdf/2014/julho/22/BoletimEpidemiol\%2D\%2Dgico-Influenza-SE28.pdf

12. Brazil. Ministério da Saúde. Secretaria de Vigilância em Saúde. Influenza: monitoramento até a semana epidemiológica 25 de 2015 [Internet]. Brasília (DF); [201-?] [cited in 2015 Jul 25]. (Boletim epidemiológico) Available from: http://portalarquivos2.saude.gov.br/images/pdf/2015/julho/07/BoletimEpidemiol\%2D\%2Dgico-Influenza-SE25-2015\%2D\%2D2-pdf

13. Álvarez-Lerma F, Marín-Corral J, Vilà C, Masclans JR, Loeches IM, et al. Characteristics of patients with hospital - acquired influenza a (H1N1) pdm09 virus admitted to the intensive care unit. J Hosp Infect. 2017;95:200-6.

14. Garnacho-Monteiro J, León-Moya C, Gutiérrez-Pizarraya A, ArenzanaSeisdedos A, Vidaur L, et al. Clinical characteristics, evolution, and treatmentrelated risk factors for mortality among immunosuppressed patients with influenza a (H1N1) virus admitted to the; intensive care unit. J Crit Care. 2018:48:172-7.

15. Rodríguez-Rieiro C, Carrasco-Garrido P, Hernández-Barrera V, Andrés A, Jimenez-Trujillo I, et al. Pandemic influenza hospitalization in Spain (2009): incidence, in-hospital mortality, comorbidities and costs. Human Vaccines \& Immunotherapeutics. 2012;8:443-7.

\section{Ready to submit your research? Choose BMC and benefit from:}

- fast, convenient online submission

- thorough peer review by experienced researchers in your field

- rapid publication on acceptance

- support for research data, including large and complex data types

- gold Open Access which fosters wider collaboration and increased citations

- maximum visibility for your research: over $100 \mathrm{M}$ website views per year

At BMC, research is always in progress.

Learn more biomedcentral.com/submissions 